\title{
Postictal Magnetic Resonance Imaging Changes Masquerading as Brain Tumor Progression: A Case Series
}

\author{
Anastasie M. Dunn-Pirio ${ }^{a}$ Santoshi Billakota ${ }^{b} \quad$ Katherine B. Peters $^{a, b}$ \\ ${ }^{a}$ Department of Neurosurgery, Duke University Medical Center, Durham, N.C., USA; \\ ${ }^{b}$ Department of Neurology, Duke University Medical Center, Durham, N.C., USA
}

\section{Keywords}

Seizure $\cdot$ Brain tumor $\cdot$ Meningioma $\cdot$ Pineoblastoma $\cdot$ Magnetic resonance imaging

\begin{abstract}
Seizures are common among patients with brain tumors. Transient, postictal magnetic resonance imaging abnormalities are a long recognized phenomenon. However, these radiographic changes are not as well studied in the brain tumor population. Moreover, reversible neuroimaging abnormalities following seizure activity may be misinterpreted for tumor progression and could consequently result in unnecessary tumor-directed treatment. Here, we describe two cases of patients with brain tumors who developed peri-ictal pseudoprogression and review the relevant literature.

(C) 2016 The Author(s) Published by S. Karger AG, Basel
\end{abstract}

\section{Introduction}

Seizure is a common clinical manifestation of brain tumor with $35-70 \%$ of patients experiencing seizures during the disease course [1,2]. Transient brain magnetic resonance imaging (MRI) abnormalities are sometimes observed following seizure activity and can be mistaken for other intracranial abnormalities including tumor progression. Here, we report two cases of patients with primary brain tumors who underwent postictal brain MRIs that 


\section{Case Reports in Oncology}

Dunn-Pirio et al.: Postictal Magnetic Resonance Imaging Changes Masquerading as Brain Tumor Progression: A Case Series

were initially interpreted as concerning for tumor progression, but were ultimately determined to be sequelae of recent seizure activity. In addition, we explore the relevant literature regarding postictal MRI abnormalities.

\section{Case 1}

A 33-year-old Caucasian woman with a history of pineoblastoma (WHO grade IV) diagnosed 2 years earlier presented to our clinic for a second opinion consultation due to concern for tumor recurrence. At the time of diagnosis, the patient was treated with subtotal resection followed by craniospinal irradiation and received an adjuvant chemotherapy regimen consisting of cisplatin, cyclophosphamide and vincristine. She had been off therapy for more than a year and was clinically and radiographically stable. However, she recently experienced a first-time seizure event consisting of two close together generalized tonic-clonic seizures resulting in a brief hospitalization and levetiracetam initiation. A brain MRI obtained 2 days following the event was concerning for left occipital leptomeningeal enhancement (fig. 1b).

Initial recommendations in light of the new enhancement were to begin further chemotherapy, but the patient sought a second opinion at our institution. Less than 1 month after the seizure, in preparation for our consultation, a subsequent MRI was obtained that demonstrated nearly complete disappearance of the previously described abnormal occipital enhancement (fig. 1c). At the time of our evaluation, the patient was at her neurologic baseline. We surmised that the transient leptomeningeal enhancement on MRI was in fact due to seizure activity rather than pineoblastoma dissemination to the leptomeninges and recommended that she remain off therapy with continued close monitoring.

\section{Case 2}

A 62-year-old Caucasian man with a recurrent left cerebellar atypical meningioma (WHO grade II) and complex partial seizure disorder is status post three tumor resections and multiple courses of chemoradiotherapy with last tumor progression occurring 1 year ago resulting in treatment with bevacizumab. Recently, the patient was admitted to the hospital for seizures in the setting of levetiracetam noncompliance. This hospitalization was complicated by refractory seizures evolving into status epilepticus. A prolonged video electroencephalogram captured multiple seizures originating from the right temporal region.

A brain MRI obtained during that admission revealed right temporo-occipital lobe leptomeningeal enhancement concerning for tumor progression (fig. $2 \mathrm{~b}$ ). However, after further review, the enhancement seemed to be confined to the parenchyma and more consistent with recent seizure activity without change in the location of the tumor itself. The patient's seizures were eventually controlled and he was discharged home on phenytoin, levetiracetam and lacosamide. A repeat MRI a month later showed near resolution of the parenchymal enhancement that was seen during the inpatient admission, thus confirming the suspicion of seizure-induced MRI changes (fig. 2c). 
Dunn-Pirio et al:: Postictal Magnetic Resonance Imaging Changes Masquerading as Brain Tumor Progression: A Case Series

\section{Discussion}

Transient brain MRI abnormalities following seizure activity have long been a recognized phenomenon [3]. Postictal MRI findings are widely variable and can be observed at the area of epileptic focus or at distant sites reflecting seizure transmission along neuronal networks [4]. A retrospective review of 26 patients diagnosed with seizure-related MR signal changes found that the most common MRI feature was increased T2 signal with some degree of mass effect. Two patients had leptomeningeal enhancement and almost half of MRIs with available diffusion-weighted imaging and apparent diffusion coefficient showed restricted diffusion. Areas of involvement included cortical and subcortical structures and in one instance involved the cerebellum. Fifteen patients had complete resolution of MRI abnormalities, but 11 experienced only partial resolution with residual gliosis or volume loss. It was also noted that status epilepticus was associated with a higher rate of incomplete resolution. On average, reversibility was documented at 62 days (range: 15-150 days) [5]. These results were consistent with prior reports of transient seizure-associated imaging findings.

Only a select number of cases in the literature specifically address seizure-associated imaging changes in the setting of primary brain tumors. Similar to our patient examples, Finn et al. [6] in 2007 reported two patients with gliomas who previously had undergone resection and chemoradiation and later developed seizures with postictal transient MRI findings that mimicked tumor progression. In addition, they discussed a third patient with a parietal meningioma previously treated with surgery, radiation and hormonal therapy, whose postictal MRI was initially worrisome for an infection based on clinical history. All three cases had reversible meningeal/gyral enhancement on MRI with near to total resolution on follow-up imaging. More recently, a French group in 2011 published a small retrospective analysis examining 10 brain tumor patients who were all previously treated with surgery and radiotherapy and had a history of seizure with transient postictal MRI abnormalities without evidence of tumor progression [7]. Interestingly, they found that all patients shared a similar pattern of radiographic abnormality following the seizure episode, consisting of focal cortical and/or leptomeningeal enhancing lesions. They coined this phenomenon 'peri-ictal pseudoprogression.'

One of the conclusions drawn from the retrospective study was that contrary to the published observations of transient postictal MRI changes in patients without tumors who most often exhibit focal cortical T2 hyperintensity and mass effect secondary to edema, postictal MRIs of brain tumor patients may be more likely to demonstrate cortical/leptomeningeal enhancement. This was certainly the case in our two cases and in the case series discussed above. This radiographic pattern may be useful to help distinguish tumor progression from postictal findings. Another inference from the study was that because all 10 patients had undergone radiotherapy, perhaps prior radiation treatment resulted in vascular changes leading to increased blood brain barrier breakdown susceptibility following seizure activity, causing enhancement. Our two patients were both treated with radiation as well as the other cases discussed. This is an interesting hypothesis, but with a high incidence of brain tumor patients receiving radiation therapy, as well as the very low number of reported peri-ictal pseudoprogression cases, it is still difficult to make a firm association.

In conclusion, our cases reiterate the importance of recognizing that for patients with brain tumors, not all new brain MRI abnormalities represent tumor progression. Knowledge that seizures can cause transient MRI findings that may mimic tumor progression can prevent unnecessary, cancer-directed treatment and therefore we recommend repeat imaging prior to modifying treatment strategies. In our own clinical experience, when this situation 


\section{Case Reports in Oncology}

Dunn-Pirio et al.: Postictal Magnetic Resonance Imaging Changes Masquerading as Brain Tumor Progression: A Case Series

has occurred and the patient is otherwise neurologically stable, we have repeated neuroimaging 1 month after the seizure event.

\section{Statement of Ethics}

The authors have no ethical conflicts to disclose.

\section{Disclosure Statement}

Dr. Dunn-Pirio and Dr. Billakota declare no conflicts of interest. Dr. Peters participates on the advisory boards of Agios and Novocure and receives research support for Agios, Amgen, Eisai, Genentech and Merk.

\section{References}

1 Maschio M: Brain tumor-related epilepsy. Curr Neuropharmacol 2012;10:124-133

-2 Hildebrand J, Lecaille C, Perennes J, Delattre JY: Epileptic seizures during follow-up of patients treated for primary brain tumors 2005;65:212-215.

3 Kramer RE, Lüders H, Lesser RP, Weinstein MR, Dinner DS, Morris HH, et al: Transient focal abnormalities of neuroimaging studies during focal status epilepticus. Epilepsia 1987;28:528-532.

Cole AJ: Status epilepticus and periictal imaging. Epilepsia 2004;45(suppl 4):72-77.

Cianfoni A, Caulo M, Cerase A, Della Marca G, Falcone C, Di Lella GM, et al: Seizure-induced brain lesions: a wide spectrum of variably reversible MRI abnormalities. Eur J Radiol 2013;82:1964-1972.

Finn MA, Blumenthal DT, Salzman KL, Jensen RL: Transient postictal MRI changes in patients with brain tumors may mimic disease progression. Surg Neurol 2007;67:246-250.

7 Rheims S, Ricard D, van den Bent M, Taillandier L, Bourg V, Désestret V, et al: Peri-ictal pseudoprogression in patients with brain tumor. Neuro Oncol 2011;13:775-782. 


\section{Case Reports in Oncology}

\begin{tabular}{l|l}
\hline Case Rep Oncol 2016;9:358-362 \\
\hline $10.1159 / 000447350$ & $\begin{array}{l}\text { @ 2016 The Author(s). Published by S. Karger AG, Basel } \\
\text { www.karger.com/cro }\end{array}$ \\
\hline
\end{tabular}

Dunn-Pirio et al.: Postictal Magnetic Resonance Imaging Changes Masquerading as Brain Tumor Progression: A Case Series
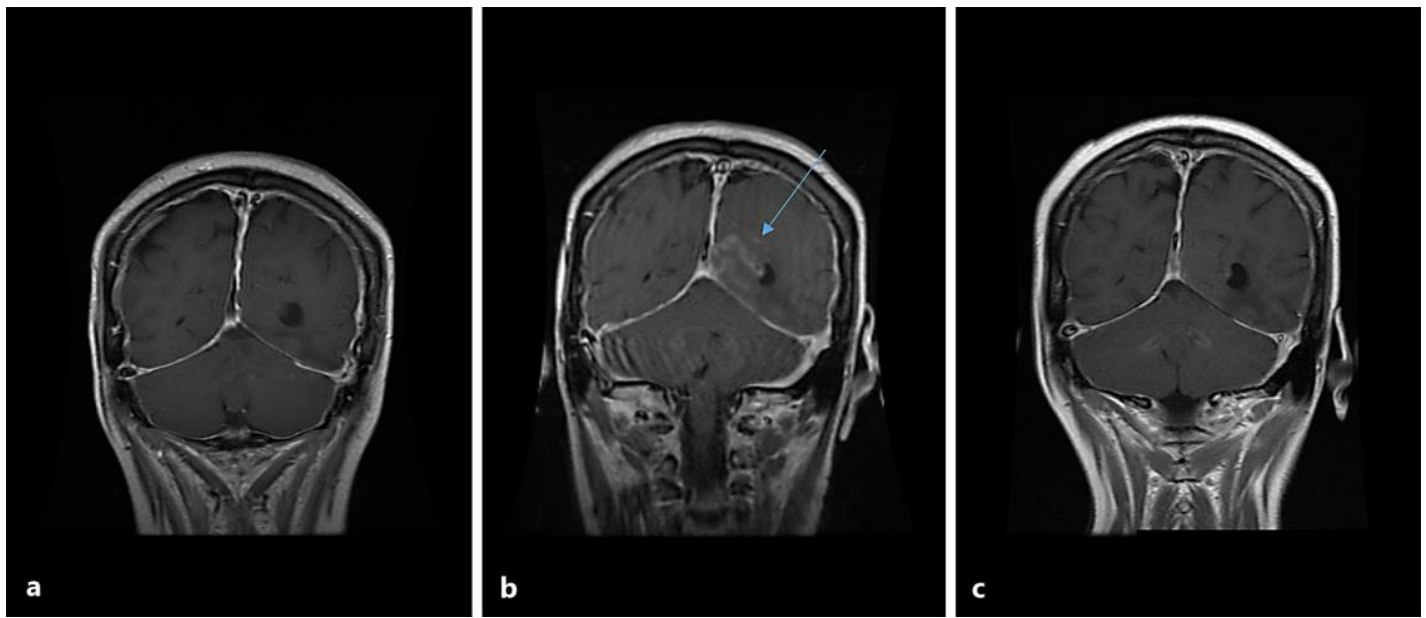

Fig. 1. Coronal brain T1+ contrast MRI images of the patient in case 1 depicting transient abnormal enhancement within the left occipital lobe 2 days following the seizure event. a MRI showing no abnormal enhancement approximately 8 months before the seizure event. b MRI showing enhancement within the left occipital lobe 2 days following the seizure event. c MRI showing resolution of left occipital lobe contrast enhancement 3 weeks after the seizure event.
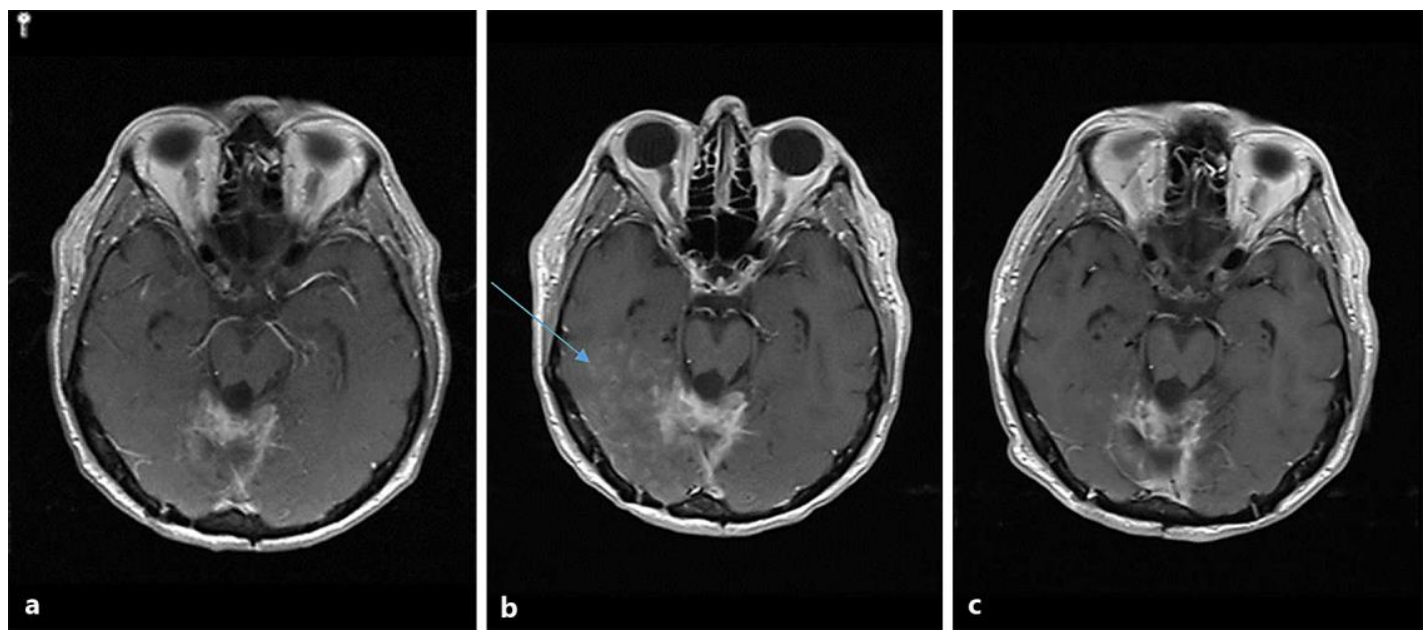

Fig. 2. Axial brain T1+ contrast MRI images of the patient in case 2 depicting transient abnormal enhancement within the right temporo-occipital lobe during the hospitalization for seizures. a Brain MRI showing stable posterior fossa enhancement 1 month prior to seizure hospitalization. b Brain MRI showing new enhancement within the right temporo-occipital lobe and unchanged enhancement within the posterior fossa during the hospitalization for seizures. c MRI showing near resolution of right temporo-occipital lobe enhancement and unchanged posterior fossa enhancement 1 month after hospitalization for seizures. 